\title{
The Role of the Speaker in Britain House of Commons
}

\author{
Ervis Iljazaj , PhD. \\ Lecturer at "European University of Tirana" (ervis.iljazaj@uet.edu.al)
}

Doi:10.5901/mjss.2015.v6n5s2p679

\begin{abstract}
The role of the Speaker of the Parliament represents a topic that constantly interests constitution and political experts, regarding both static and dinamyc profile. In this perspective, this analysis will focus on the neutral model of British parliamentary governance (speakership). As a constant reference will be used the examination of the institution of the Presidency of the Parliament in its dual role, as an internal organ of the Parliamentary Assembly, but also as a structure of high "external" institutional profile, which in collaboration with other constitutional bodies is essential for institutional qualification in which its role is located. A special attention will be paid to the form of the British government and how it has affected the powers and role of the Chairman of the Assembly in the political-constitutional order. The methodology used in this paper will be analytical. The main objective of this study will be to explain the factors that led to the installation of such model in this Country as well as causal relevance of each one. These variables such as the form of government, political system and the party system will be tested in this case for understanding the model of British parliamentary.
\end{abstract}

Keywords: Speaker, Parlament, House of Commons, Goverment form

\section{Introduzione}

II ruolo dello Speaker all'interno del Parlamento britannico non si puo' studiare a prescindere dall'evoluzione storica che l'organo rappresentativo ha avuto durante la storia politica di questo paese.

Giovanni Ferrara (1965), autorevole studioso della tematica, in un suo celebre scritto evidenzio' che "all'assemblea nella società inglese, alla funzione che essa esercita nella vita della comunità e alla forza che essa esprime corrisponde alla posizione dello Speaker, all'origine della sua nomina, all ruolo che esercita nelle relazioni internazionali con gli altri soggetti e alla forza politica e giuridica che da lui e' emanata"(p.1).

Il Presidente della Camera dei Comuni , tuttavia, non ha sempre beneficiato di un importanza uguale durante la storia del parlamento inglese. Esso ha gradualmente assunto il ruolo dell'autentico tutore delle prerogative di quest'ultimo, e del garante della regolare limitazione, da parte della Camera bassa ( la House of Commons), dei diritti dei cittadini , da iniziale, semplice porta-parole del Legislativo (Plucknett, 1960).

Lo Speaker ha costituito una testimonianza del conflitto tra gli organi costituzionali durante i secoli della modernità. Come conclusione di questo processo è l'adeguamento delle esigenze di indipendenza parlamentare a contingenze storico-politiche peculiari. Si deve precisare che la Speakership inglese ha dovuto necessariamente depurarsi da tanti iniziali vincoli per poter assumere finalmente alla terzietà magistratuale (Sturlese, 1963).

\section{La Posizione dello Speaker Durante la Storia}

I primi Speaker che addivennero a ricoprire questa carica, certamente non avevano l'indipendenza e l'imparzialita odierna . Erano considerati come Servants of the Crown, deputati che manifestavano la "volonta sovrana" ed a ottenere dalla Camera legislative medievale alcuni poteri fra i quali: l'esazione tributaria straordinaria da parte di fasce sociali crescenti. Lo Speaker operava come portavoce della Corona, ovverro collettore di coungiunzione tra il Sovrano e Parlamento (Laundy, 1964).

Dal momento in cui i Comuni iniziarono le loro riunioni separatamente dal Re e dalla sua smaller Curia, si avvertì l'esigenza di un autonomo porta-parole. In questo modo, si interrompe quella fase costituzionale nella quale i rappresentanti delle Contee e Borghi, giuridicamente mandatari delle rispettive collettività territoriali, venivano convocati sporadicamente dal Sovrano al fine di "acconsentire alla tassazione straordinaria", con la formazione di un'opinione pubblica favorevole, di fornire alla stessa informazioni sulle molteplici situazioni locali e di facilitare l'amministrazione

\footnotetext{
"This article is part of my PhD thesis: "Different models of the Parliamentary Presidency". University of "La Sapienza", Rome.
} 
regia (lacometti, 2001).

Considerando l'onerosità della funzione di rappresentanza e la debolezza iniziale dell'organo parlamentare, non suscita stupore che il portavoce dell'assemblea non fosse neppure membro della stessa, in taluni casi. Essendogli semplicemente affidato il compito di comunicare le relative deliberazioni del Sovrano, così come che non la presiedesse, né ponesse questioni da dibattere.

Il soggetto che la deteneva questa carica era vincolato dall'obbligo di rispondenza ai desideri reali - attraverso il coinvolgimento assembleare - ma anche alla Camera, circa le modalità di notifica al Monarca delle decisioni 'parlamentari' utilizzando le necessarie abilità relazionali e diplomatiche. Una carica così congegnata non poteva non essere ritenuta 'ingrata', legata da un rapporto di "doppia dipendenza" (Laundy, 1984).

La tendeziale riluttanza verso l'assunzione del nobile ufficio e giustificata dunque, con la perpetuazione consetudiniaria-seppur con rilevenati attenuazioni-fino all'epoca attuale. Questo e successo con formule di diniego 0 dichiarazioni di indegnità. In quel periodo potevano acquisire note di drammaticità per la persona che che era costretta all'esercizio di una funzione sgradita, considerato secondo Sturlese (1963) "pericolo per la stessa incoluminità fisica del suo titolare" ( p.40).

Analizando tali elementi, si comprende come il processo della prerogativa di selezione dello Speaker senza ingerenze della Corona si sia estrinsecato in modo lentamente graduale. Solo con il terminare del Regno degli Stuart e possibile individuare Speaker ritenuti Servants of the House (e non più esclusivamente of the Crown), che proclamino come loro dovere precipuo l'attività "into the House". Gli speaker precedenti benché nominate dal Monarca non sono stati sempre un docile strumento a sua disposizione. II periodo medievale e post-medievale è caratterizato di figure che hanno contribuito con la loro coragiosa pervicacia, all'incremento delle competenze della Camera dei Comuni, garantendo l'affermazione di un potere di indirizzo-controllo della Camera bassa nei confronti dell'Esecutivo (Limon \& Mc Kay, 1997).

In tal senso, esempi emblematici sono rappresentati dal gesto di Peter de la Mare, nominato anche Spokeman del Good Parliament nel 1376 . Consistente in una forte critica nei confronti dell'avarizia nella spesa pubblica da parte della Corona, ed in un rifiuto, manifestato 'in nome della Camera', alla concessione di nuovi tributi senza una rimozione in modo preventivo agli ministri corrotti invisibilmente. II potere di impeachment che avrebbe avuto tanto rilievo nell'ordinamento costituzionale future ${ }^{1}$.

Questo episodio, certificò la possibilità di un una importante influenza politica dello Speaker nei confronti della propria camera. L'affermazione della spettanza alla House of Commons della deliberazione delle spese pubbliche si riconnette a Sir. John Guildesborough, il quale ricoprì l'ufficio nel 1380. Questo orientamento dopo permetterà di consolidare le prerogative della Camera, subordinando la concessione delle imposte all'accoglimento delle iniziative legislative, presentate originariamente sottoforma di claims. In materia finanziaria ( Money Bills) ai Comuni diretti da Thomas Chaucer vienne riconosciuta la iniziativa esclusiva da comunicare alla Corona dopo la obligatoria approvazione dei Lords (Biscaretti Di Ruffia, 1998). I Tudor tentarono di depotenziare e di utilizzare le Camere come strumento di legittimazione del proprio potere dinastico, dopo la fase della contesa politica vissuta dal Parlamento nel corso della guerra delle Due Rose ( Laundy, 1972).

La scelta di Speakers ossequientemente collegati al Sovrano era aderente al mutato contesto istituzionale. II processo di affrancamento della figura dalla nomina reale subiva una profonda battuta d'arresto. Speakers fortemente devoti alla Sovrana e consapevoli della presenza di una serie di materie sottratte alla deliberazione assembleare, si rinvennero anche in tale fase. Questo perche la Camera non si riuniva a cadenze definite ma, era soggetto alla convocazione regia in quanto "function of monarchy"( Mcllwain, 1990).

La dinastia Tudor dichiarandosi parte importante del potere legislativo, cercò sostegno nel Parlamento , in modo da ricevere riconoscimento da esso in termini di legittimità.

L'assemblea poteva, comunque, fruiva di una cospicua autonomia, almeno sotto il profilo organizzatorio, per le ragioni sopra menzionate. Nonostante ancora collegata strutturalmente alla Corona, la carica di Speaker, era già avvolta in un'aura di prestigio, ritenendosi la tappa di un cursus honorum destinato a concludersi nella carriera giurisdizionale (Limon \& Mc Kay, 1997).

Alla vigorosa e rivendicazione dei privilegi della Camera e all'abilità nella conduzione dei lavori parlamentari corrisponde in quel periodo anche l'autorevolezza dell'ufficio dello Speaker. Propio in questo periodo da parte dei Comuni emerse la profonda consapevolezza per quanto riguarda la importanza della indipendenza del loro Presidente

\footnotetext{
${ }^{1}$ Si noti come l'istituto in oggetto, nato con finalità penalistiche ed intimamente correlato all'inesistenza di un rapporto fiduciario tra Governo e Parlamento, consenti alla Camera di bilanciare il potere regio di scioglimento assembleare, cosi iscrivendo nella forma di governo inglese due poteri reciprocamente equilibrati.
} 
soprattutto dalla Corona. La scelta degli argomenti nella formulazione dell'ordine del giorno delle sedute prima di tutto necessitava l'assicurazione di una compiuta e diffusa libertà di epsressione (Laundy, 1984).

L'inizio dell'arte di gestione del dibattito parlamentare, si manifesto durante il regno di Elisabetta I (1558-1603). II protagonista fu Edward Coke, giurista di fama, Speaker nel periodo considerato. Con una condiscendenza nei confronti dei "desiderata" della Sovrana, egli ha sostenuto l'idea della nomina dello Speaker da parte della stessa con la giustificazione da non sottrare alla Camera inutile risorse mentali e temporali nell'elezione di un personaggio non gradito alla Corona. Dimostrando notevoli capacità proceduriali spesso manipolava ed alterava i processi decisionali interni alla Camera. Favoriva l'aprovazione parlamentare di provedimenti graditi alla Corona mediante raffinati ed artati rinvii, ostacolando il dibattito e la votazione di atti ad essa ostili (Cardon, 1883).

In ogni caso, e' opportuno riconoscere al Presidente Coke un ruolo di assoluta influenza nella concreta evoluzione di questo istituto. Proprio in relazione alle sue capacità relazionali e diplomatiche, da questo momento sarà impossibile prescindere per esercitare la funzione direttiva. Con l'affermazione della dirimente regola procedurale del "casting vote", oggi solenne norma consuetudinaria invalsa in molti parlamenti europei, terminò il periodo elisabettiano. In virtù di questa norma, allo Speaker è preclusa la non partecipazione alle votazioni, salvo il caso di divisione paritaria dell'aula nella deliberazione (Keir, 1961).

Le prerogative del Parlamento risultarono fortemente compresse e ridimensionate, durante la dinastia reale degli Stuart (1603-1714), determinando una elevazione all'ufficio direttivo dell'Assemblea di soggetti collaterali al Sovrano, 0 comunque ad esso subordinati (Laundy, 1972).

Nel 1621 il Re Giacomo I invitò lo Speaker in carica a rivolgersi alla Camera con una dichiarazione dove si sosteneva che qualsiasi attività di controllo ne confronti del Governo regio o dei ministri dello Stato doveva essere sospeso. Questi "antiche ed indubbi diritti ed eredità" parlamentari nella prospettiva sovrana si consideravano come "concessioni", espressione della mera tolleranza mostrata dai precedenti Monarchi (lacometti, 2001).

Dopo una condizione di involuzione e limitazione delle prerogative della Camera bassa, i Comuni si dimostrarano non più disposti a subire questi limitazioni nei confronti della Corona e nell uso delle loro tradizionali garanzie. II celebre Committee of the Whole House, un comitato infra-assembleare, assunse una rilevanza importante, al fine di dibattere senza formalità ed in assenza dello Speaker ( Lovell, 1962).

I deputati assunsero la consapevolezza della importanza dei meccanismi e i strumenti procedurali e della pericolosità in un loro uso non adeguato. Propio per questo si rivendicarono una spazio di intangibilità parlamentare sottratto alle manipolazioni di uno Speaker eccessivamente solidale con il Re. II potere di aggiornamento delle sedute che si e' esercitato artatamente avrebbe inibito la continuità e libertà delle liberazioni e un esempio significativo (Keir, 1961).

Il regno di stuart nonostante gli elementi sopra menzionati, rappresentò una tappa importante verso l'affermazione del potere di nomina di Speakers indipendenti dalla Corona. Questo ha resso palese il desiderio di afrancamento dei Comuni da qualsiasi vincolo ostativo della supremazia del Legislativo.

Solo con il regno di Carlo II si approfondisce la discussione necessaria per la neutralità ed imparlzialità dello Speaker. I Comuni sancirono il loro inalienabile diritto di scegliere, in totale autonomia, il proprio Presidente, in una controversa disputa relativa al mancato conferimento dell'assenso reale alla proposta assembleare di nominare all'ufficio direttivo Edwar Seymour (Lovell, 1962).

L'ambiguità parzialmente ridotta della consacrazione delle prerogative parlamentari non e stata risolta da questo episodio. Quando il neo eletto Speaker Williams si praclamò soggetto al Sovrano ed ai Comuni si manifesto tutto la discutibile ruolo dello Speaker (Lloyd, 1976).

Sebbene in modo incompleto e relativo, la appena emersa indipendenza dello Speaker dalla Corona, ha potuto vedere fermarsi il proprio percorso di definitivo consolidamento a causa di un sopravvenuto fattore, il condizionamento operato dalle fazioni politiche nell'esercizio dell'ufficio medesimo. Tra i secoli XVII-XVIII apparvero prepotentemente nella società civile britannica alcuni rassemblement, costituiti da uomini politici sotto la penetrante egida di magnati e famiglie nobiliari aristocratiche di estrazione terriera, al fine di supportare interessi privati o settoriali nei confronti dei pubblici poteri ( Bailey, 1910).

Dove la divisione politica era fondata su motivi di ordine eminentemente religioso, rispetto al periodo elisabettiano - la contrapposizione che si realizzò tra tali groups o connexions, riproduceva gli stessi orientamenti esistenti nella valutazione dei rapporti Corona-Parlamento. Proprio su questa disputa ebbe genesi un embrionale sistema partitico dicotomico, formato, durante il Regno di Carlo II, Abhorrers ed Addressers, e, successivamente, Tories e Whigs (Mortati, 1973).

II contesto partitico, si definì con maggiore nitidezza solo a seguito della Glorious Revolution (1688-1689). Iniziò il consolidamento di una attività assembleare di natura oppositoria rispetto al Governo in carica. Erano i prodromi della 
transizione che la forma di governo monarchico costituzionale pura, avrebbe conosciuto nel Settecento, segnando inevitabilmente le tappe di una correlata modificazione della posizione e del ruolo dello Speaker nell'ordinamento ( Laundy, 1972).

L'esponente Whigs Henry Powle, II Presidente parlamentare - 0, più precisamente, della Convention effettuò la solenne investitura dei nuovi Sovrani -, denominato First Commoner, in modo da evidenziare plasticamente la supremazia della Camera sugli altri organi costituzionali. La Speakership sarebbe assurta a prestigiosi livelli di indipendenza anche dai raggruppamenti partitici, nella prima metà del secolo XVIII, grazie alla meritoria opera di Arthur Onslow, eletto alla carica nel 1728 ( Laundy, 1972).

Dotato per le questioni procedurali, Onslow, utilizzò la sua influenza all'interno del Partito per imporre l'equilibrio e la garanzia della stabilità e dell'equilibrio all'interno dell'assemblea. Onslow si servì delle preesistenti parliamentary practices, forgiando nuove Rulings, da poter controllare le intemperanze delle opposte compagini politiche, e tutelare le minoranze dalle pressioni governative, interpretando in modo attivo il suo ruolo (Andonis, 1976).

Per questa ragione, è definito come il primo Speaker "impartial servant of the House" dalla giuspubblicistica britannica. Tuttavia, nel secolo successivo, si sarebbe avvertita pienamente la necessità di Presidenti d'Assemblea svincolati dai condizionamenti partitici, specialmente da quelli provenienti dai gruppi determinanti per la sua ascesa all'ufficio.

Con Charles Manners-Sutton (Tory), eletto alla carica nel giugno 1817, si percepì il bisogno di allontanare tali soggetti dal partito che ne avevano favorito la nomina. L'ultimo esempio di partisan Speaker, benché il suo operato sia ritenuto non pregiudizievole dell'autonomia parlamentare e della imparzialità, è considerato James Abercromby, il suo successore ( Laundy, 1964).

La neutralità e terzietà della posizione presidenziale, associata all'indefettibile ruolo ordinatorio del dibattito assembleare e di tutela delle minoranze, quale affermatosi attraverso una graduale - ma inesorabile - evoluzione storico-giuridica, si realizza con l'inizio del secolo XIX.

Come si evince dalla pregressa analisi della storia costituzionale e parlamentare britannica, l'indipendenza dello Speaker dalla Corona rappresenta il background del carattere dell'imparzialità. È possibile asserire che questa peculiarità di equidistanza abbia mutato la propria connotazione nel corso dei secoli, traslando da una originaria nozione di esercizio indipendente della funzione direttiva nei confronti di ingerenze "esterne", verso una concezione più ampia, contemplante sia i condizionamenti esogeni che quelli endogeni - ossia infraassembleari -all'espletamento del mandato presidenziale.

L'indipendenza "esterna" dell'organo monocratico si impose grazie alla sopravvenuta rilevanza delle funzioni di porte-parole nei riguardi del Sovrano, riconosciute originariamente all"oratore ufficiale" dell'Assemblea.

Secondo Ferrara ( 1969) , "il Presidente si stagliava come elemento unificante la collegialità della Camera prima di esercitare compiti di direzione ed organizzazione del dibattito "interno". Come tale, soggetto beneficiante di una crescente grado di autonomia nella assunzione delle decisioni, soprattutto rispetto alle contrapposizioni esistenti fra le fazioni o partiti rappresentati in Parlamento" ( p. 191).

Mediante la presentazione al Monarca delle deliberazioni camerali e delle istanze emerse nel suo ambito, il potenziale di rivendicazione da egli espresso, costituì un influente fattore di coagulazione degli orientamenti politici propugnati dalle componenti "interne", garantite dalla accresciuta autorevolezza del proprio Presidente, in modo singolare e collettivo Corollario dell'indipendenza strutturale e funzionale strenuamente richiesta dal potere parlamentare- I' imparzialità dello Speaker- non consente una formulazione esaustiva di questa figura, in quanto ha esercitato una determinante incidenza la sua funzione "materialmente giurisdizionale" sulla piena affermazione della stessa nell'architettura istituzionale inglese ( lacometti , 2001).

La portata della nozione di fairness della Presidenza d'Assemblea nel sistema britannico, si esplica attraverso quest' ultimo concetto. Essa si mostra come un genus contemplante necessariamente a due species: l'espletamento di compiti sostanzialmente magistratuali e la neutrale indipendenza da qualunque legame politico, partitico o relazionale. Intraprendere la prosecuzione di quel percorso storico iniziato nei paragrafi precedenti, pare opportuno per individuare l'inscindibile legame.

Come già rilevato, il principale compito assegnato allo Speaker per numerosi secoli consisteva nell'interpretazione e conseguente applicazione del diritto parlamentare inglese, il cui fondamento, in evidente analogia con le fattezze del sistema giuridico di common law, ha prevalente natura consuetudinaria ed è forgiato dalla regola giurisprudenziale dello stare decisis (Torre, 2000).

II Presidente poteva essere reputato un giudice tra giudici, dovendo garantire effettività a norme principalmente non scritte attraverso un rinvenimento del 'precedente' cui conformare l'operato dei deputati, talora procedendo all'integrazione creatrice di diritto mediante proprie risoluzioni, in guisa da garantire un tempestivo adeguamento delle procedure a nuove esigenze, anche se originariamente i parlamentari effettuavano una attività paragiurisdizionale, 
applicando il 'diritto comune' ed integrandolo talvolta con lo Statute Law (Criscuoli, 2000).

Le regole di organizzazione e funzionamento della Camera erano imperniate sulla "traditional Practice"- ossia una serie di norme consolidatesi a partire dal XVII secolo, quando si accingeva a divenire principio caratterizzante della forma di governo la preminenza delle Camere rispetto al potere regio, fino al 1832- in cui si realizzò la fondamentale riforma elettorale (Reform Act), che ristrutturò i collegi di rappresentanza, contribuendo alla modernizzazione dell'apparato garantistico e democratico dell'ordinamento ( lacometti, 2001).

Questa prassi, disciplinando l'attività parlamentare emulando i procedimenti deliberativi delle Corti medievali ecclesiastiche, rese indispensabile la presenza di un soggetto che, dirigendo i dibattiti interni, compisse anche un'opera interpretativa dell'antico corpus normativo al fine di consentire l'esercizio del proprio diritto di parola ad ogni componente interno (Manetti, 1990).

Tale opera innovativa si tradusse nelle "Rulings from the Chair", fonte diversa, ma parimenti importante, del diritto parlamentare inglese, consistente nell'insieme delle regole promananti della Presidenza ad integrazione delle consuetudini. II compito venne svolto in modo non totalmente dissimile da quello caratteristico della magistratura di common law, mediante una interpretazione rispettosa del precedente, e talora integrativa dello stesso, ma comunque ispirata ad un criterio di ragionevolezza ( Torre, 2000).

Come il buon senso è reputato essenziale requisito per la integrazione-creazione del common law, così la ragionevolezza doveva costituire paramento di riferimento dell'attività latamente "normativa" dello Speaker ( Floridia, 1986).

La stessa dottrina britannica, la quale paragona il soggetto in esame ad un giudice, definendone le Rulings come case-law, perché sorto dalla risoluzione di controversie concrete di diritto parlamentare, sottolinea la natura materialmente giurisdizionale delle prerogative dell'organo monocratico dell'Assemblea. Esse si pongono in rapporto di specificazione-integrazione non solamente verso l'ancient Practice, ma anche nei confronti delle norme procedurali di nuova formazione, gli Standing Orders (Wilding \& Laundy, 1972).

Questi ultimi - definiti dall'autorevole letteratura in materia come "direttive che la Camera ha tramandato nei tempi al fine di mettere a punto la prassi, di modificarla o di introdurre nuove regole o procedure" - sono stati predisposti a partire dal 1832, ossia nel momento in cui, variando il rapporto tra classe politica e società civile - scaturente dall'ampliamento della rappresentanza parlamentare - si avvertì l'esigenza di approntare norme procedurali che assicurassero al Governo la possibilità di controllare l'Assemblea, al fine di attuare il proprio indirizzo programmatico vieppiù copioso ed articolato ( Floridia, 1986).

I precedenti parlamentari, la famigerata Practice, risultavano possedere una profonda vocazione garantisticaessendosi sviluppati contestualmente alla Camera- mirante alla prioritaria tutela delle minoranze e dei singoli deputati anche a pregiudizio delle funzionalità operative dell'aula ( Jennings, 1957).

La finalità degli Standing Orders, che riuscirono a garantire decisionalità senza caducare la base consuetudinaria delle regole procedurali, ma, al contrario, codificandola e integrandola, fu proprio questa. L'adozione di moderne fonti scritte di diritto parlamentare non eliminò però l'inossidabile ruolo assolto dall'attività interpretativa ed applicativa di natura giurisdizionale appannaggio dell'imparziale Speaker, benché essa avesse visto la propria genesi in assenza di una formalizzazione scritta del relativo tessuto normativo ( lacometti, 2001).

II nuovo assetto istituzionale poneva la necessità della presenza di un soggetto che esercitasse una penetrante opera di influenza e di orientamento nella evoluzione delle stesse norme regolamentari, promuovendone l'introduzione positiva da parte dell'assemblea, ed applicandole secondo criteri di idonea interpretazione ed equità, soprattutto nelle ipotesi di sollevazione di un "point of order" da parte di un singolo componente il collegio ( Hanson \& Wiseman, 1962).

II compito di evitare le eccessive lungaggini dilatorie del dibattito in aula, e di introdurre poteri disciplinari in capo alla speakership idonei a consentire efficienza ad una Camera sempre più eterogenea politicamente e socialmente, venne assegnato agli Standing Orders. La facoltà oratoria di ciascun parlamentare doveva essere circoscritta. Tale elemento importante appariva connessa all'idea secondo la quale dalla discussione in aula possa scaturire una deliberazione, e quindi un risultato tangibile, solo se è canalizzato entro regole tecniche gestite da un organo razionale imparziale.

Arthur Onslow si adoperò per l'individuazione delle disposizioni procedurali, imponendone l'osservanza dopo una adeguata interpretazione, ed attribuendo una particolare cogenza al precedente, nel sistema britannico (Laundy, 1972) . Durante la presidenza di Shaw-Lefevre, eletto all'indomani del Reform Act 1832, e, soprattutto, di Henry Bouverie William Brand, Speaker dal 1872, cui va ascritta la risoluzione di una delle impasse più intense della storia della House of Commons si realizzò una radicale trasformazione di questo specifico strumento parlamentare. Nel 1881 venne introdotta la "chiusura della discussione" al fine di combattere l'ostruzionismo dei nazionalisti irlandesi - endemico in questa delicata fase delle loro relazioni con la Madrepatria - ed approvare rapidamente alcuni progetti di legge. Grazie all'attività 
di 'buoni uffici' esercitata dall'autorità direttiva dell'assemblea, persuasa della inevitabilità di un simile istituto per garantire la funzionalità di una Camera che, a causa della eccessiva elasticità delle proprie disposizioni procedurali, era stata oggetto di un atteggiamento dilatorio da parte delle forze fra cui, quelle che lottavano contro l'Union Act 1801, atto costitutivo del Regno unito di Gran Bretagna ed Irlanda - ostili alla adozione di determinate deliberazioni che potessero penalizzare le rappresentate istanze ( Wilding \& Laundy).

La mozione che doveva consentire tale interruzione del dibattito fu modificata, fra il 1881 ed il 1887, assegnando il potere di presentazione della stessa a singoli communers e non più al Presidente. Quest'ultimo poteva ricusare la votazione qualora avesse riscontrato discrepanze con i diritti della minoranza ( Laundy, 1972).

La competenza dello Speaker in materia di controllo e selezione delle richieste di caducazione della discussione permane intatta, anzi, se possibile, rafforzata, in guisa da evitare abusi dell'istituto posti in essere non per esigenze di economia procedurale, bensì per ragioni strumentali.

Cocludere però queste preliminari note sull'evoluzione storica della speakership anglosassone, senza citare l'operato del Presidente Arthur Wellesley Peel - in carica dal febbraio 1884 e quasi impossibile, il quale, promosse la introduzione di novellati Standing Orders idonei a limitare ab origine le ore disponibili per lo svolgimento delle sedute, attraverso la predisposizione di forme automatiche di aggiornamento delle stesse ( lacometti, 2001).

\section{I Poteri dello Speaker}

Le fonti principali che sancivano i poteri dello Speaker nell'ordinamento britannico sono state per lungo tempo le norme di procedura e i precedenti parlamentari. Ma con l'adozione delle norme regolamentari - a partire dalla prima metà del secolo XIX - e stato reso possibile una razionalizzazione ed un ampliamento dei poteri e facoltà della speakership inglese, confermandone, altresì, la sua natura sostanzialmente giurisdizionale (lacometti, 2001).

È opportuno precisare però sin da ora che - come rilevato da uno dei maggiori esperti di procedure parlamentari britanniche - l'analogia tra le funzioni dello Speaker e quelle di un giudice di common law non risulta idonea ad annullarne le notevoli differenze sotto il profilo sia strutturale che concretamente operativo ( Tosi, 1993).

II Presidente, infatti, molto spesso non beneficia delle medesime guarentigie riconosciute ad un magistrato per l'espletamento dei propri compiti, ad esempio - ma non esclusivamente - in materia di tempo assegnato per la deliberazione e di possibilità di ascoltare ripetutamente, ed in dettaglio, le affermazioni delle parti contrapposte, e quindi di riesaminare il caso. Qualora sorga una controversia all'interno dell'aula, esso deve dirimerla con la massima prontezza possibile, senza necessità di allegare alle proprie 'declaratorie' motivazioni eccessive 0 ultronee, per finalità di risolutezza ed efficienza nei riguardi della membership parlamentare ( Tosi, 1993).

Quindi, si può sostenere che allo Speaker - malgrado tutte le differenziazioni e precisazioni testé esposte - siano riconosciuti rilevanti attribuzioni, esercitate con notevole discrezionalità, relative alle modalità della discussione e delle votazioni, operando esso in tale ambito - sicuramente più ristretto ma senz'altro meglio definito rispetto a quello in cui agiscono altre Presidenze di assemblea - con la massima autorità, in ossequio alla nota espressione "The Speaker like the Pope is infallible", formulata da alcuni esponenti della dottrina (Ameller, 1967).

L'organizzazione dei lavori parlamentari è demandata, invece, al Cabinet, il quale è fornito dei mezzi idonei alla formulazione dell'ordine del giorno delle sedute, ossia della individuazione delle materie da esaminare e sulle quali deliberare. Procediamo allora nella analisi delle diverse funzioni attribuite al Presidente all'interno Camera dei Comuni, iniziando da quelle derivanti dalle norme di procedura parlamentare, gli Standing Orders già richiamati.

Innanzi tutto, in base allo S.O. n. 313, esso è abilitato a richiedere al Chairman of the Ways and Means di presiedere, in particolari circostanze, l'assemblea, senza alcuna comunicazione formale alla stessa.

Inoltre, lo S. O. n. 4 gli conferisce il potere di nomina, per ciascuna sessione, del cd. Chairmen's Panel, composto da soggetti deputati ad espletare l'incarico di Presidenti temporanei di Commissioni, nonché dal Chairman of Ways and Means e dai suoi Vice. Le altre molteplici norme regolamentari secondo lacometti (2001) possono essere distinte in :

1. giudizi di ammissibilità preventivi al dibattito;

2. facoltà che ne consentono il più efficiente e snello svolgimento. Nella prima categoria sono annoverabili:

a) il potere di dichiarare l'inammissibilità di progetti di legge e mozioni. Ad esempio, lo Speaker potrebbe precludere la presentazione di un Money Bill redatto in modo non conforme alle regole procedurali (S.O. n. 23) ;

b) la facoltà di selezionare gli emendamenti relativi ad una mozione 0 ad un progetto di legge, attraverso la correlata possibilità di richiedere al proponente una delucidazione sulle finalità dello strumento oggetto del giudizio, prima di adottare una decisione definitiva (S.O. n. 32) . Nell'esercizio di questa attribuzione, il Presidente si ispira a determinati criteri, non considerando ammissibili, ad esempio, gli emendamenti 
riproduttivi di altri, garantendo la discussione di quelli che consentono di disciplinare in modo plurimo il medesimo oggetto, ovvero, in caso di presentazione di proposte di modificazione di analogo contenuto, accordando il privilegio dell'esame a quelle contrassegnate da una maggiore esaustività ed intelleggibilità nella formulazione;

c) la delicata competenza in materia di selezione degli oratori ammessi alla discussione. In virtù di una radicata consuetudine, lo Speaker deve concedere diritto di parola al primo deputato che, elevandosi dal proprio scranno, attiri la sua attenzione, secondo la nota formula "To catch the Speaker's eye" . Nell'ipotesi di richiesta contestuale da parte di più commoners, allora la prelazione dovrà esser riconosciuta al decano per età, ovvero ad esponente del Cabinet rispetto ad uno dell'opposizione, o, ancora, ad un front-bencher piuttosto che ad un back-bencher (Boulton, 1989).

Peraltro, la prassi recente i è incaricata di mostrare come sia invalso l'uso di chiedere al Presidente il diritto di intervento nel dibattito prima dell'inizio dello stesso - attraverso l'invio di personali missive che gli consentano di comprendere l'utilità della istanza e l'effettivo interesse alla partecipazione da parte del richiedente - in guisa da garantire all'organo monocratico la possibilità di ripartire il tempo a disposizione tra le varie componenti politiche secondo un criterio di alternanza tra maggioranza ed opposizione, ovvero tra i diversi collegi elettorali rappresentati ( Lloyd, 1976).

Inoltre, nel corso degli ultimi anni si è sviluppato un trend teso a favorire un rapido espletamento di tale compito, grazie alla sottoposizione al banco della Presidenza - da parte dei Chief Whips - di liste di oratori che i rispettivi Leader dei gruppi intendono coinvolgere nella discussione. Ma queste Whip's Lists hanno generato forti perplessità e critiche proprio fra quei parlamentari di retroguardia - cd. backbenchers - che le reputano limitative delle loro potenzialità d'espressione - in quanto ispirate sovente, nella stesura, da un principio gerarchico - ed inoltre pregiudizievoli per la discrezionalità dello Speaker . E' opportuno precisare però che, benché ogni Presidente sia gravato dall'obbligo di garantire una partecipazione il più possibile numerosa alle discussioni assembleari, l'esiguità temporale non consente sempre la soddisfazione di tutte le esigenze di intervento manifestate, in ossequio al principio generale che tende a promuovere ed inverare - a nostro parere inevitabilmente - la decisionalità dell'organo parlamentare (Lloyd, 1976)

d) L'insindacabile giudizio relativo alla ammissibilità delle interrogazioni ( S.O. n. 21-22); compete infatti allo Speaker escludere la formulazione delle questions non afferenti ad una sfera di responsabilità ministeriale, talora attraverso il previo interpello del destinatario governativo della stessa al fine di verificarne la congruità ratione materiale. È questa una competenza di notevole importanza e complessità, anche a causa della esistenza di cd. supplementary questions, sorte in via eccezionale all'inizio del XX secolo e confermate dalla prassi, che, per la loro non preventiva conoscibilità, rappresentano uno tra gli strumenti ispettivi più temuti dai componenti del Cabinet; concretamente, si configurano come repliche dell'interrogante alla risposta fornita dal Ministro in sede di question time, che consentono al primo la richiesta di ulteriori chiarimenti, anche contemplanti domande integrative; pur tuttavia, l'esercizio di questa facoltà, non delineando un diritto tout court de deputato, deve essere autorizzato dalla Presidenza ( Laundy, 1972). Nella seconda categoria di attribuzioni della speakership si collocano, invece:

a) il potere inerente alla legittimità delle richieste di chiusura anticipata della discussione - la cd. Ordinary closure (S.O. n. 36) - che, nate per finalità preminenti di natura antiostruzionistica, sono prevalentemente presentate da un singolo deputato della maggioranza per consentire al Governo di ridurre i tempi del dibattito ed accedere così immediatamente alla votazione di determinati provvedimenti. È prerogativa dello Speaker, appunto, verificare che tale istanza non sia lesiva dei diritti delle minoranze o delle regole camerali.

b) Le sanzioni disciplinari, di contenuto materialmente giurisdizionale, che si sostanziano in strumenti idonei a garantire all'organo monocratico la direzione e direzione del dibattito, evitandone la portata dilatoria, attraverso il richiamo verbale del deputato inadempiente o prolisso (S.O. n. 42), l'allontanamento dalla seduta (S.O. n. 43), e, nelle ipotesi più gravi, la richiesta di interdizione dai lavori dell'aula per un determinato intervallo temporale (generalmente non superiore ai venti giorni di seduta 0 all'intera durata della sessione), sulla quale, però, è chiamata a pronunciarsi la Camera (S.O. n. 44) ( Norton, 1991).

Le attribuzioni appena enucleate, come detto, trovano fondamento sia negli Standing Orders, sia nella prassi parlamentare appositamente codificata ed innovata. A tali fonti però si affiancano espresse disposizioni legislative che prevedono ulteriori funzioni e responsabilità in capo al Presidente - $c d$. Statutory Duties - consistenti in compiti di natura interpretativa e certificativa ( Laundy, 1972) 
Esempi in questo ambito sono espressi dal potere dello Speaker di individuare e dichiarare un Bill come di natura finanziaria, con tutte le conseguenze in termini di procedimento legislativo previste dai Parliament Acts ( 1911 - 1949). Nella selezione di tali progetti di legge, la dottrina si mostra pressoché univoca nel ritenere che il parametro di giudizio della Presidenza debba essere costituito dal precedente, ad evidente corroborazione della tesi sulla natura materialmente magistratuale del suo ufficio direttivo ( lacometti, 2001).

Infine, a competenze di matrice notificatoria deve essere ricondotta la individuazione, nei casi di ambiguità 0 incertezza, del Leader dell'opposizione parlamentare di Sua Maestà che, in virtù dell'art. 10 del Ministers of The Crown Act ( 1937) beneficia di una periodica indennità da parte dell'erario, qualora venga qualificato come tale dallo Speaker attraverso una "decisone scritta ed inappellabile ove sorgessero dubbi riguardo al partito che abbia maggior seguito (ovviamente, dopo quello espressione del Cabinet, ndr) o alla persona riconosciuta come Leader". Questa specifica attribuzione ben si coniuga con il ruolo di indefettibilità istituzionale accordato all'opposizione parlamentare nella dinamica della forma di governo britannica ( De Vergotinni, 1980).

\section{La Neutralità e le Ragioni Giustificative}

Dopo aver tentato, nei paragrafi successivi, una coerente sintesi relativa alle peculiarità strutturali e funzionali del fascinoso istituto in esame, appare ineludibile, in conclusione di capitolo, interrogarsi sulle ragioni poste a fondamento di una speakership di tal fatta, come detto, sostanzialmente magistratuale e connotata da una dose pressoché assoluta di imparzialità.

Per espletare questa operazione è doveroso considerare, come fattori determinanti :

1. l'estensione del suffragio;

2. il tendenziale e strutturato bipartitismo del sistema politico;

3. la forma di governo e la sua evoluzione ( lacometti, 2001)

Con riferimento al primo elemento, si sottolinea come solo tra la fine del XVIII e l'inizio del XIX secolo si addivenne a quella profonda riforma in materia di composizione territoriale dei collegi elettorali e di accesso alla rappresentanza sia sotto il profilo dell'elettorato attivo che passivo - mediante il già richiamato Reform Act 1832, che, associata all'introduzione di nuove norme di procedura parlamentare, rese necessaria la presenza di un soggetto superpartes, disciplinatore del dibattito interno ad una assemblea sempre più eterogenea, e dunque potenzialmente instabile, sotto il profilo socio-politico ( lacometti, 2001).

La tendenziale compattezza della maggioranza filo-govenativa e, nel contempo, la sopravvenuta consapevolezza della imprescindibilità della funzione dell'opposizione sollecitavano, pertanto, la preposizione alla carica di una personalità assolutamente scevra da condizionamenti 'di parte'. Solo in tal modo la minoranza avrebbe potuto esercitare il ruolo di leale alternativa al Governo in carica, e lo Speaker medesimo avrebbe assicurato decisionalità e democraticità alla Camera ( Tosi, 1993).

La rappresentanza unitaria dell'assemblea, al di sopra delle eventuali divisioni al suo interno, era stata resa possibile dall'esistenza, almeno sino alla fine del secolo XVIII, di un comune "nemico" - come afferma icasticamente Tosi (1993 ) nel citato saggio in materia -, "questa "politicità" esterna del Presidente era bilanciata da una corrispettiva "apoliticità interna" all'Assemblea, consentendo il riconoscimento nella sua posizione di terzietà da parte degli attori politico-partitici: il ruolo, dunque, di organo portatore delle istanze comuni della Camera dinanzi al Re permise lo stabilirsi del carattere di imparzialità, intesa come "neutralità nei riguardi dei contrasti interni alla assemblea, in nome di una solidarietà creatasi per far fronte ad uno scontro considerato più importante" (p.527). Conquistata la vittoria da parte del Parlamento, veniva meno la necessità di una rilevanza "esterna" dello Speaker, ma ciò non pregiudicò la preservazione del suo carattere di asetticità rispetto all'agone politico.

Il fenomeno in esame è esplicabile tenendo conto del secondo e terzo elemento della nostra classificazione, ossia l'assetto bipartitico della Assemblea inglese e la conformazione dei "mutevoli rapporti fra i supremi organi costituzionali in relazione alla funzione di indirizzo politico" ( Lanchester, 2002).

Infatti, la formazione progressiva di un sistema partitico caratterizzato dalla presenza di due partiti rilevanti dapprima Tories e Whigs, ed oggidì Conservative e Labour - ha accentuato l'importanza della neutrale centralità del Presidente dell'Assemblea, deputato a garantire effettività a qosiuel principio di "alternanza" che, assieme alla responsabilità dell'Opposizione di 'Sua Maestà' ed alla formula elettorale maggioritaria a turno unico di tipo plurality, ha consentito alla forma di governo inglese di dispiegare stabilità, trasparenza e funzionalità ( Bulmer \& Tomas, 1967).

Le relazioni costruttivamente antagonistiche fra i due maggiori Partiti, fondate sull'accettazione di un comune background assiologico, permettono il perpetuarsi di un organo ritenuto imparziale dalle parti, che riflette la condivisione sul "metodo parlamentare", 
ossia sulla riproduzione istituzionale "dell'unanimità tra i partiti sui temi sociali e politici fondamentali" . Ma, come rilevato, il consolidamento di una posizione presidenziale così configurata non sarebbe stato possibile senza la trasformazione della sovranità parlamentare nella forma di governo in una effettiva ed intensa preminenza del Gabinetto, ed in esso, del Primo Ministro ( Tosi 1993).

L'evoluzione ha condotto la Camera a divenire la sede di ratifica degli indirizzi governativi, che, nel contempo, appropriato luogo di dibattito e di dialettica fra maggioranza ed opposizione, realizzando, in tal modo, un progressivo affrancamento dello Speaker da ogni possibile subordinazione o condizionamento partitico.

È parimenti noto come il processo di spoliticizzazione della speakership sia stato favorito dalla progressiva affermazione di altri organi connotati politicamente, impedendo così che il direttore del dibattito si tramutasse in un selezionatore degli argomenti oggetto dello stesso.

La dottrina più autorevole sottolinea come la scelta del soggetto cui affidare la competenza relativa a quegli atti che incidono sulla politica legislativa delle Camere - quali, in primo luogo, quelli attinenti alla formulazione dell'ordine del giorno - è normalmente coerente con la forma di governo, concorre a connotarla, ed è intimamente connessa con la posizione ed il ruolo assegnato al Presidente dell'assemblea (Tosi, 1993).

Quest'ultimo, come già rilevato, nell'esperienza inglese non è mai stato titolare del potere di formazione dell'ordine del giorno di seduta, rimesso dapprima - nella fase precedente all'insediamento dell' attuale forma di governo monistica a prevalenza dell'Esecutivo - al Primo ministro, e successivamente - segnatamente dalla metà del secolo XIX -, al Leader of The House, componente della compagine ministeriale ( Jenninigs, 1957). Esso possiede il principale compito di controllare la Camera grazie all'ausilio dei Whips, dopo aver definito l'ordine del giorno attraverso adeguate 'intese' con l'opposizione condotte "behind the Speaker's Chair" , e di vigilare sulla attuazione dell'indirizzo politico-legislativo del Governo ( Torre, 2000). La presenza di convenzioni informali tra le componenti dell'assemblea sulle tematiche oggetto dell'attività camerale estromette, pertanto, il Presidente dalla dinamica della politica attiva, confermandone la assoluta terzietà anche nell'epoca contemporanea.

\section{Biblografia}

Adonis, A. (1993). Parliament Today. Manchester-New York: Manchester University press.

Bailey, S.D. (1971). British parliamentary democracy (III ed). London-Toronto, Greenwood Press.

Boulton, C.J. (1989). Treatise on the Law, Privilegies, Proceedings and Usage of Parliaments, (XXI ed) . London, Butterworths.

Bulmer-Tomas, I.( 1967). The Growth of the British Party System, (I, $2^{\circ}$ ed). Baker.

Cardon, R. (1883). Svolgimento storico della Costituzione inglese. Torino. Loescher.

Criscuoli, G. (2000). Introduzione allo studio del diritto inglese, Le fonti, (III ed). Milano, Giuffrè.

De Vergottini, G. (1980). Opposizione parlamentare, in "Enciclopedia del Diritto", (Vol. XXX). Milano.

Ferrara, G. (1965) . II Presidente di assemblea parlamentare. Milano, Giuffrè.

Floridia, G.G. (1986). II regolamento parlamentare nel sistema delle fonti. Milano, Giuffrè.

Hanson, A.H. Wiseman, H.V. (1962). Parliament at work. London. Stevens \& Sons Ltd

Jennings, I. (1957) . Parliament. Cambridge. Cambridge University Press.

lacometti, M. ( 2001). I Presidenti di Assemblea Parlamentare. Milano, Giuffrè .

Keir, D.L. (1961). The Constitutional History of Modern Britain since 1485. London. Black

Lanchester, F. (2002). Le costituzioni tedesche da Francoforte a Bonn. Introduzione e testi. Milano, Giuffrè.

Laundy, P. (1984). The office of Speakers in the Parliaments of Commonwealth. London. Quiller Press.

Laundy, P. ( 1964). The Office of Speaker. London. Cassell

Limon, D.,Mc Kay,W.R. (1997). Treatise on the Law, Privilegies, Proceeding and Usage of Parliament, (XXII ed). London. Butterworths.

Lloyd, S. ( 1976). Mr. Speaker Sir. London. Jonathan Cape.

Lovell, C.R. (1962). English Constitutional and Legal History. A Survey. New York. Oxford University Press.

Manetti, M. (1990). La legittimazione del diritto parlamentare, Milano, Giuffrè,

Mcllwain, C.H. (1990). Costituzionalismo antico e moderno. Bologna, II Mulino.

Mortati, C. (1973). Le forme di governo. Padova, Cedam.

Norton, P. (1991). The House of Commons. Cambridge. Cambridge University Press.

Plucknett, T. F. ( 1960). English Constitutional History from the Teutonic Conquest to the Present Time. London.

Sturlese, L. ( 1963). II Re e i Lords nel Parlamento medievale inglese. Milano. Giufre'

Torre, A. (2000). Il magistrato dell'assemblea, Saggio sui presidenti parlamentari. Torino, Giappichelli.

Tosi, S.( 1993). Diritto parlamentare. Milano, Giuffrè.

Wilding, N., Laundy, P. ( 1972). An Encyclopedia of Parliament. London. Littlehampton Book Services Ltd 